Illinois State University

ISU ReD: Research and eData

Theses and Dissertations

4-13-2021

\title{
Feeling the Light: Sensory Substitution Devices in Research and Rehabilitation
}

Stephanie Nicole Hartling

Illinois State University, hartlings23@outlook.com

Follow this and additional works at: https://ir.library.illinoisstate.edu/etd

\section{Recommended Citation}

Hartling, Stephanie Nicole, "Feeling the Light: Sensory Substitution Devices in Research and Rehabilitation" (2021). Theses and Dissertations. 1438.

https://ir.library.illinoisstate.edu/etd/1438

This Thesis is brought to you for free and open access by ISU ReD: Research and eData. It has been accepted for inclusion in Theses and Dissertations by an authorized administrator of ISU ReD: Research and eData. For more information, please contact ISUReD@ilstu.edu. 


\section{FEELING THE LIGHT: SENSORY SUBSTITUTION DEVICES IN RESEARCH AND REHABILITATION}

\section{STEPHANIE N. HARTLING}

\section{Pages}

The ecological approach to perception-action seeks to understand an individual's control of goal-directed actions by focusing on the relationship or fit between animal and environment (Turvey, 2019). This fit determines what behaviors are possible for that animal - what Gibson (1979/2014) referred to as affordances. Affordances emerge from this fit between the animal and the environment; however, this fit is malleable such as with the addition of a tool. Tools can modify a person's ability to perceive affordances. Tools such as sensory substitution devices (SSDs) utilize one modality (such as touch) when another modality is unavailable or compromised (such as in individuals who are visually impaired) to allow the user to perceive and act within their environment. In this thesis, I plan to make and support two claims about SSDs:

1) SSDs can be used to perceive and act on affordances in the context of performing everyday behaviors, such as navigating from place to place and 2) since SSDs can be used to perceive affordances, the devices can be used in rehabilitation therapies for people who are (or have become) visually impaired or "balance-impaired". Furthermore, I will explain why SSDs and why one SSD in particular - the enactive torch (ET) - should be incorporated into other fields of research (specifically, rehabilitation). 
KEYWORDS: sensory substitution; sensory substitution device; Enactive Torch; ecological psychology; functional therapeutics 
FEELING THE LIGHT: SENSORY SUBSTITUTION DEVICES IN RESEARCH AND REHABILITATION

\author{
STEPHANIE N. HARTLING
}

\begin{abstract}
A Thesis Submitted in Partial Fulfillment of the Requirements for the Degree of MASTER OF SCIENCE

Department of Psychology

ILLINOIS STATE UNIVERSITY
\end{abstract}


Copyright 2021 Stephanie N. Hartling 
FEELING THE LIGHT: SENSORY SUBSTITUTION DEVICES IN RESEARCH AND REHABILITATION

STEPHANIE N. HARTLING

COMMITTEE MEMBERS:

Jeffrey B. Wagman, Chair

Peter J. K. Smith 


\section{ACKNOWLEDGMENTS}

I would like to thank Cody (Christopher) Atkinson, Julie Campbell, and Louis H. Favela for their suggestions on this thesis. I would also like to thank my parents, Priscilla and Robert Hartling Jr., and brother, Robert Hartling III, for their support.

S. N. H. 


\section{CONTENTS}

Page

ACKNOWLEDGMENTS

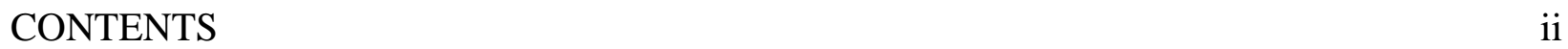

FIGURES

CHAPTER I: AN ECOLOGICAL APPROACH TO UNDERSTANDING PERCEPTION 1

An Overview of Ecological Psychology 1

$\begin{array}{ll}\text { Perception in Action } & 4\end{array}$

$\begin{array}{ll}\text { Sensory Substitution Devices } & 6\end{array}$

CHAPTER II: USING TOOLS IN A MEANINGFUL ENVIRONMENT 10

$\begin{array}{ll}\text { What Tool Use is in Ecological Psychology } & 10\end{array}$

Tools as extensions of action capabilities $\quad 12$

Learning in Tool Use $\quad 13$

$\begin{array}{ll}\text { Ecological Interfaces } & 15\end{array}$

$\begin{array}{ll}\text { Vibrotactile Flow in SSD } & 17\end{array}$

$\begin{array}{ll}\text { Sensory Substitution Devices as Tools } & 20\end{array}$

CHAPTER III: ADDRESING CALIM 1: SSDS IN PERCEPTION AND ACTUALIZING

$\begin{array}{ll}\text { AFFORDANCES } & 23\end{array}$

Sensory Substitution Devices (SSDs) Review 24

Perception of Environmental Properties Using SSDs 29

Perception of Affordances Using SSDs 31

$\begin{array}{ll}\text { Summary } & 33\end{array}$

CHAPTER IV: ADDRESSING CLAIM 2: SSDS IN REHABILITATION 34 
A Function-Based Approach to Rehabilitation 34

Tool Use in Therapeutics $\quad 36$

SSDs Outside of Laboratory Research 38

$\begin{array}{ll}\text { Summary } & 42\end{array}$

CHAPTER V: EVALUATION OF THE ENACTIVE TORCH AND FUTURE

$\begin{array}{ll}\text { DIRECTIONS } & 44\end{array}$

The Enactive Torch $\quad 44$

The Fit of the ET for Rehabilitation $\quad 44$

$\begin{array}{ll}\text { Future Directions } & 47\end{array}$

$\begin{array}{ll}\text { In Conclusion } & 49\end{array}$

$\begin{array}{lr}\text { REFERENCES } & 50\end{array}$ 


\section{FIGURES}

Figure

1. Reflecting Light 2

2. Converging Light 2

$\begin{array}{ll}\text { 3. Probing by foot } & 7\end{array}$

4. The Enactive Torch $\quad 8$

5. Tool Use and Perception 11

6a. Obstruction of Visual Field 18

6b. Obstruction of Visual Field with SSD 18

7. Inverse Shadow Effect 46 


\section{CHAPTER I: AN ECOLOGICAL APPROACH TO UNDERSTANDING PERCEPTION}

\section{An Overview of Ecological Psychology}

What perception is and what is perceived. Perception can be defined as one's awareness of one's surroundings. Such awareness allows for the control of goal-directed behaviors, thus creating a relationship between the environment and the organism. The ecological approach to perception states that (1) perception is of this relationship (or fit) between the animal and their environment and (2) the purpose of perception is to guide action according to this fit (Turvey, 2019). This fit determines what behaviors are possible for the animal - what Gibson (1979/2014) referred to as affordances. Given that animals have many different action capabilities and environments have many different properties, there are always multiple possible behaviors in any given context (Wagman et al., 2018). Affordances, then, are possibilities for behavior that emerge from the multifaceted relations between properties of the animal and the environment. They are activity-specific meanings for a specific animal (Turvey, 2019; Wagman, 2020).

The information for perception. Gibson (1979/2014) proposed that information about this multifaceted fit between animal and environment is available in the lawfully structured energy arrays available at a point of observation (Thomas et al., 2020). The information for visual perception, for example, is available in the ambient optic array - the structured reflected light that surrounds an animal (Gibson, 1979/2014; Thomas et al., 2020; Turvey, 2019). Light becomes structured when it reflects off of substances and surfaces in the environment eventually converging to a point of observation occupied by an animal (see figure 1 and 2). It is by means of this structured reflected light at a point of observation that animals not only perceive properties of surfaces in their environment but also their relation to those surfaces (the animal-environment 
2 relationship) (Thomas, Riley, and Wagman, 2020). Thus, we do not perceive light per se.

Rather we perceive by means of light.

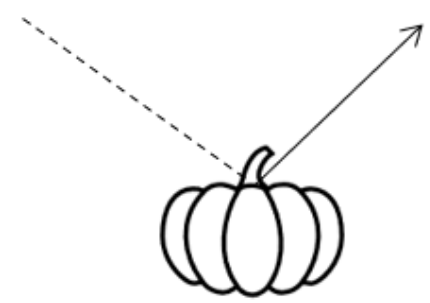

A
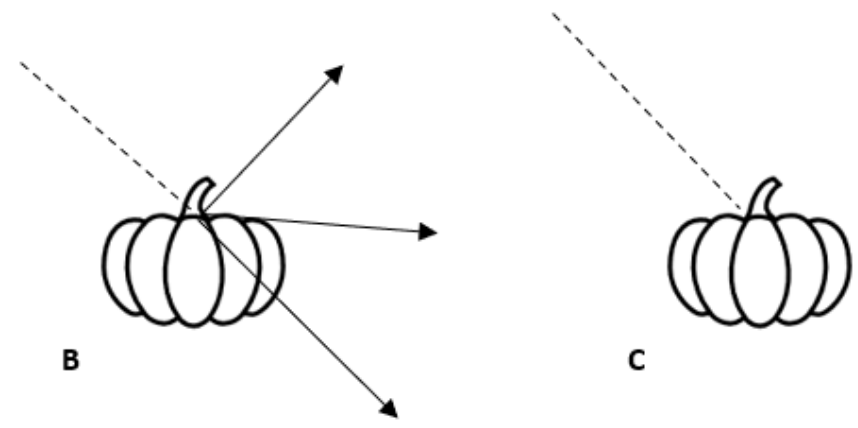

C

Figure 1 Reflecting Light: Light can reflect off of surfaces and objects in three ways: it can bounce off the object or surface at the same angle it struck it (A), it can bounce off in different directions (B), or it can be absorbed into the object or surface (C).

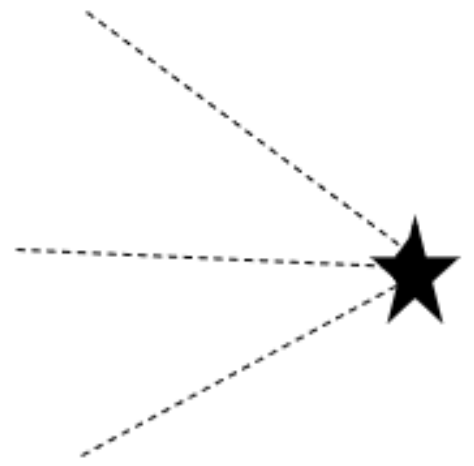

Figure 2 Converging Light: Light converging at a point of observation. 
Perception by means of light occurs within the optic array and with optic flow. The optic array is structured light that surrounds the organism. Optic flow is systemic changes within the optic array that occur when surfaces move with respect to an organism or when the organism moves with respect to the surface. In other words, optic flow is the pattern of the changing optical texture caused by the movement between the organism and their environment (Burton and Radford, 1978; Warren and Strelow, 1985). Optic flow stimulation is important in perception of movement, but also for perception of shapes, distance and movement of objects, and for control of the organism's movement (Royden and Moore, 2012). The ambient optic array and optic flow are both important for the perception of affordances as they specify (changing) relationships between the animal and the environment.

Gibson states that perception is not representational or computational; rather, perception is the detection of information (in structured energy arrays, like the optic array) that is lawfully related to meaningful, complex, and emergent relationships between the perceiver and their environment (Gibson 1979/2014; Wagman 2020). Some relationships in the optic array change and others remain constant. The ones that remain constant are known as invariants (i.e. optic flow is an invariant because it specifies movement regardless of speed, direction, etc.). It is these invariants that are relevant to affordances (Turvey, 2019). In particular, the ecological approach focuses on this invariant information generated by movement in exploration of the environment (Gibson 1979/2014). Invariant patterns in structured energy encountered at a point of observation provides information about the possible relationships between the organism and the environment (Wagman, 2020). Thus, patterns of reflected light at a point of observation provides information about affordances. 


\section{Perception in Action}

Affordances depend on changing action capabilities. The fit between action capabilities and environmental properties is determined, in part, by factors such as body size, strength, flexibility, and experience in given task (Cole et al., 2013; Adolph, 2019). However, given that all of these factors change over both short and long-time scales, the fit between animal and environment is malleable (changes over time). For example, as infants grow, their body capabilities change, eventually affording behaviors such as crawling and eventually walking. Furthermore, environmental cues can also alter affordances for the infant, such as parental cues (holding the child's hands to teach them how to walk) (Adolph, 2019). The action capabilities that afford infants crawling are not the same action capabilities that afford infants walking; therefore, infants must develop new action capabilities when they learn to walk. This shows that perception is dynamic, or rather, adapts and changes over time as our bodies change and we gain experience. This further suggests that the relationship between the organism and the environment is also dynamic.

Infants undergo relatively rapid changes in strength, balance, and coordination over the scale of weeks. Infants learn to create and detect information for affordances in the moment, meaning they perceive affordances based on what they can currently do with their body and their current skills in their environment (Adolph, 2019). For example, infants new to crawling attempt to crawl down slopes that are too steep, but experienced crawlers do not. What's more interesting is that this learning does not transfer between crawling and walking: experienced crawlers who learned to walk attempted to walk down slopes too steep, just as when they first learned to crawl (Adolph, 2019). This is due to the differences in the two behaviors in terms of posture, balance, coordination, and point of observation in the optic array. Infants have to re-learn what slopes 
they can walk down because walking provides a new set of affordances due to the new strength and flexibility their body has once walking, compared to when they were crawling.

The previous example shows that changes in strength, balance and coordination alter the fit between action capabilities and the environment, thus altering affordances; however, affordances are also dependent on body size and the environment (such as fitting a hand through varying aperture sizes). A study by Ishak et al. (2008) investigated whether, when, and how participants chose to reach through an aperture of varying sizes. In experiment 1, participants were asked to use their dominant hand to reach through apertures of varying sizes to retrieve small target objects. Participants scaled their reaching behavior to their hand size, meaning participants chose to reach through the aperture based on the size of their hand (if they determined their hand was small enough to fit through the aperture, they reached through it). In a follow up experiment, participants chose whether, when, and how to reach through the aperture when wearing a padded prosthetic that increased the width of their hand. The results showed that participants adjusted their reaching behavior accordingly, meaning they took into account the new size of their hand when reaching through the aperture.

The study conducted by Ishak et al. (2008) shows how the animal-environment relationship influences perception of affordances: in experiment 1, participants' natural (unaltered) hand size influenced reaching behavior. Even when this relationship was changed, as in experiment 2, participants were able to adjust their perception and behavior and reach through the aperture successfully. The study showed how affordances emerged from the relationship between the animal and the environment and how they changed when the fit changed (see Mark, 1987). 


\section{Sensory Substitution Devices}

Not only do action capabilities change over short and long-time scales but perceptual capabilities do as well. One short term change in perceptual capabilities is when a person uses an object as a perceptual tool. Tools, like the prosthesis used in the above study, alter the body's dimensions and abilities, requiring the individual to recalibrate their action decisions to consider the changing body dimensions. But tools can also change a person's perceptual capabilities. In a series of experiments conducted by Wagman and Hajnal (2014a, b), blindfolded participants were asked to probe an inclined surface with a rod held in one hand or the other, both hands in multiple grip configurations, or one foot or the other. They were asked to determine if they would be able to stand on the surface. The study found that perception of affordances for standing on the surface (being able to stand on the incline) reflected the action capabilities of the participant and was unaffected by which limb (or configuration of limbs) was used.

In another study investigating perception of affordances, participants were able to perceive properties of either the probe (length) or the probed surface (distance) by exploring (via probing or tapping) the surface with a hand-or-foot-held rod (Wagman, Hartling, \& Mason, 2020; see figure 2). The rod was attached to the foot and the participant was asked to probe the surface with the rod, and this was repeated with the participant holding the rod in their hand. The rod served as a tool for exploring the environment, allowing the individual to explore surfaces that they would not otherwise have been able to reach. 


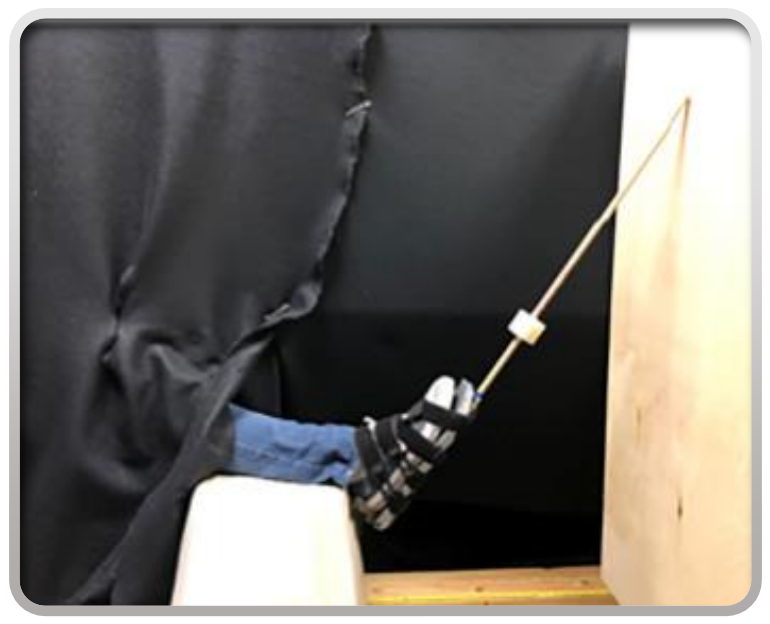

Figure 3 Probing by foot: Participants used a rod held in the hand or attached in the foot (see image) to probe a surface. Participants reported the length of the rod and the distance of the probed surface.

The rod in the examples above was used in much the same way as a long cane might be used by a visually impaired individual. Such tools are low-tech sensory substitution devices (SSDs) because they allow a person to perceive properties with one perceptual modality that they might otherwise perceive with other perceptual modalities. More high-tech SSDs transform stimulation patterns from one form (e.g., light) to another (e.g., vibration patterns) (de Paz et al., 2019; Favela et al., 2018; Maidenbaum, Abboud, and Amedi, 2014). The purpose of these devices is to provide the user information about the world when one modality is unavailable or compromised (such as in individuals who are visually impaired) to allow the user to perceive and act within their environment. SSDs, then, allow the user to detect information about their environment that they might otherwise obtain by other means under other circumstances.

Furthermore, the SSD allows the user to perform everyday behaviors within their environment, meaning that the user of the SSD perceives and acts on affordances by means of the information provided by means of the tool (SSD). For example, when using the SSD, individuals can perceive fixed apertures, such as doorways, and can use the SSD to perceive 
whether that doorway is pass-through-able (Favela et al., 2018). In a different study, blindfolded participants were able to determine how far away a surface was from them when using a different SSD, the EyeCane, (Maidenbaum et al., 2014). These studies provide evidence that blindfolded participants can use SSDs as perceptual tools to perceive affordances and environmental properties.

There are two different general sensor mechanisms that an SSD design can utilize: 1) an infrared laser that measures distances (Maidenbaum et al., 2014; Kerdegari et al., 2015; Cancar et al., 2013; Favela et al, 2018; see figure 4) and 2) a camera that creates a pixelated representation of a given object (Bird et al., 2009; Stetten et al., 2007; Horvath et al., 2014). Both kinds of sensors translate the information detected (e.g., light) into a different stimulation pattern, such as felt vibrations (Cancar et al, 2013; Froese et al., 2012) or auditory output (Maidenbaum et al., 2014). These stimulation patterns can be used to perceive properties such as distance (Maidenbaum et al., 2014) or aperture size (Favela et al., 2018).

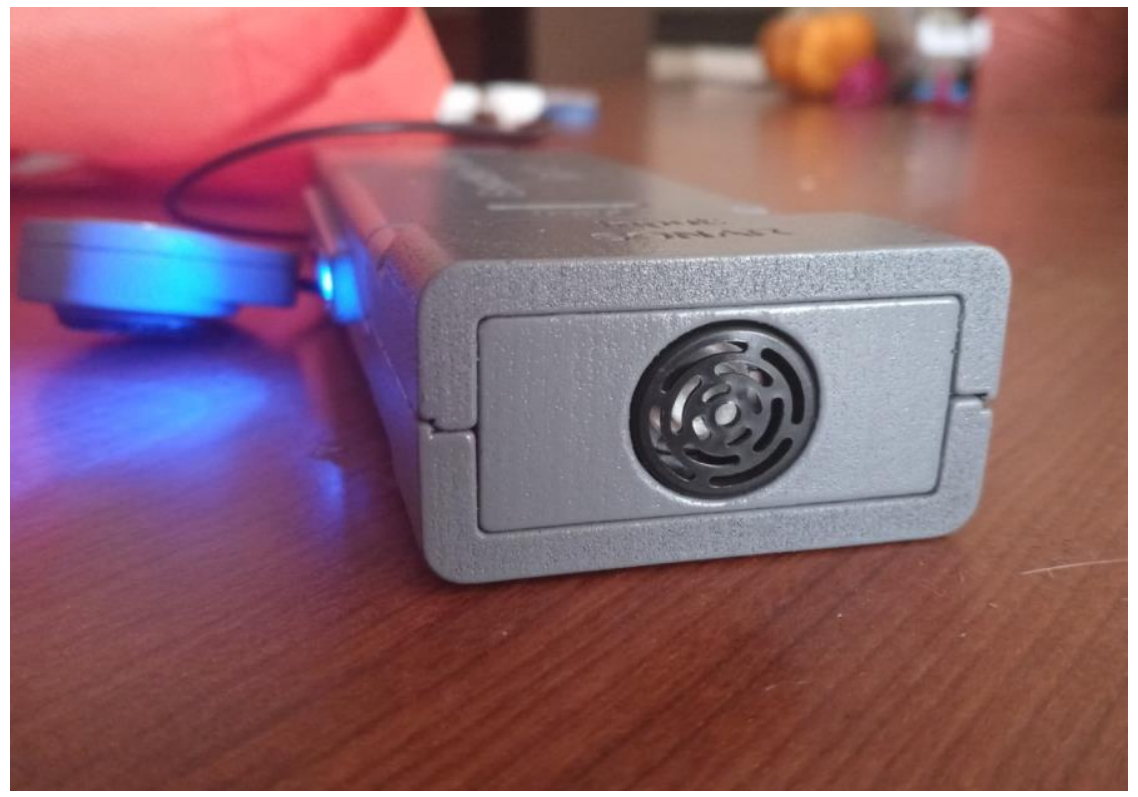

Figure 4 The Enactive Torch; A Sensory substitution device using infrared lasers to detect distance information. 
Studies have found that, while using SSDs, participants are able to successfully perceive and act on affordances such as catching a ball or walking through a door frame (Bird et al., 2009, Favela et al., 2018). SSDs, then, allow the user to perceive affordances that they might otherwise perceive by other means under other circumstances. Moreover, participants using SSDs are able to act on the same affordances as they would under other circumstances. The SSD allows the user to directly interact with their environment, meaning the user of the SSD perceives affordances and can act upon those affordances with the use of the tool (SSD). When using the SSD, individuals can perceive fixed apertures, such as doorways, or dynamic ones, such as gaps, allowing for judgment of affordances such as step-over-able when using the SSD (Favela et al., 2018). Furthermore, studies have found that judgements about passing through a doorframe (Favela et al, 2018) and distance estimates from an object (Maidenbaum et al., 2014) were significantly more accurate when participants used the SSD than with only vision. This provides evidence that SSDs enable the successful control of behavior. 


\section{CHAPTER II: USING TOOLS IN A MEANINGFUL ENVIRONEMNT}

\section{What tool use is in Ecological Psychology}

Ecological psychology focuses on a scientific explanation of the lawful perceptual control of goal directed behaviors, making the fundamental unit of analysis the animalenvironment relationship, not either in isolation (Wagman and Day, under review; Gibson, 1979/2014; Turvey, 2019). The fit between the animal and their environment is what determines what affordances are available to the animal and what goal directed behaviors the animal can perform (Wagman \& Day, under review). Moreover, it is at this level - the animal-environment relationship - that all psychological processes occur, including tool use (Wagman \& Day, under review; Gibson, 1979/2014).

Gibson (1979/2014) defines tools as graspable, portable, manipulatable, and usually rigid. Tools alter the fit between the animal and their environment by altering the perception of affordances (Wagman and Day, under review). The tool changes both the perceptual abilities (what affordances are perceivable) and the action capabilities (what affordances are available) of the animal (Wagman and Day, under review). For example, a stepping stool provides the animal with the ability to grab an object they otherwise would not have been able to reach and a flashlight provides the user a means to safely navigate their environment (see figure 5). Tool use, however, only occurs when it -the tool- is used to actualize goal-directed behavior. For example, merely carrying a fishing rod or a flashlight does not constitute tool use because the rod or flashlight are not being used for goal-directed behaviors. For the tool to alter perceptual abilities, the tool must be used to perform a goal directed behavior. 


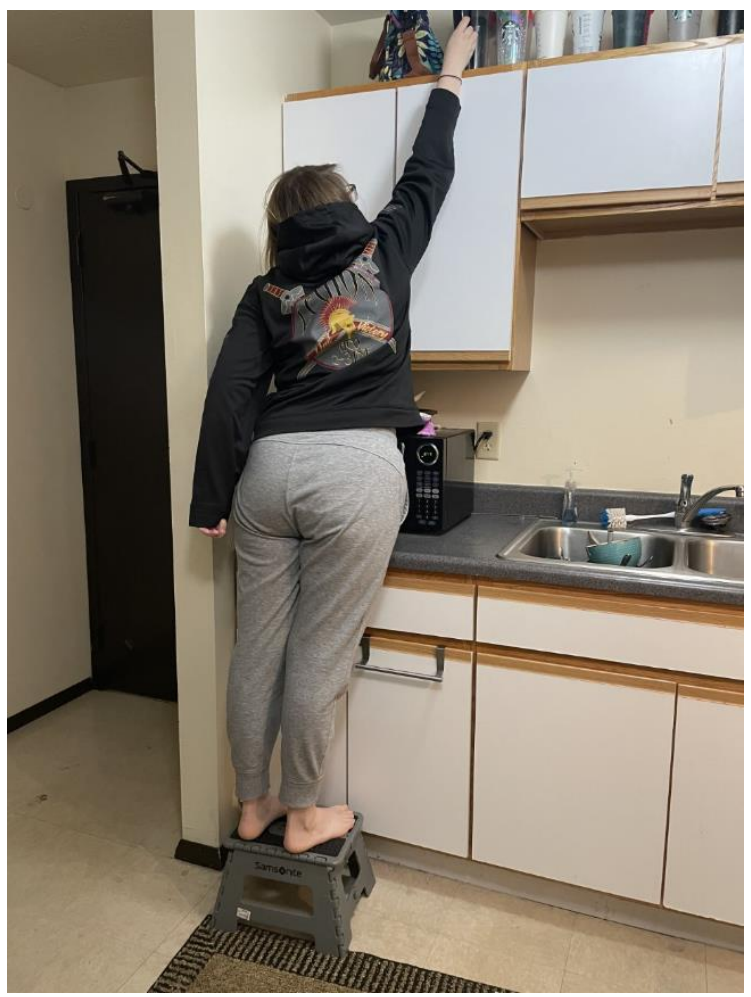

Figure 5 Tool use and perception: The stepping stool affords the individual the opportunity to reach an object they would not have otherwise been able to reach.

While tools change the perceptual and behavioral capabilities of the user, the tool itself has multiple possible uses, or affordances. For example, a screwdriver can be used to twist screws, it can be used to puncture, or it can be thrown. Exploration with a wielded tool at a point of observation provides information about the altered relationship between the animal and the environment, and in turn, it also provides information about the affordances of the tool. (Wagman and Day, under review). For example, people can perceive many different affordances of a wielded occluded object and they can perceive affordances of that object under different wielding circumstances (Hajnal et al., 2007; Wagman et al., 2017). When performing a behavior, different body parts are recruited to perceive and exploit affordances (such as the hand when wielding an occluded object, like in the above example). 


\section{Tools as extensions of action capabilities}

As previously stated, tools change the perceptual and behavioral capabilities of the user, but tools also alter our experience of our bodies by becoming a part of our body. When a tool is used, the tool is incorporated as an extension of our action capabilities, thus becoming a part of our body schemas (Pagano \& Day, 2020). Body schemas were once viewed as a relatively fixed internal model of the size, shape, and mass of the body stored in memory (Head, 1970; Iodice et al., 2015). Recently, it has been theorized that body schemas are simultaneously malleable and stable and perceived in real time, suggesting our body schemas can update 'online' (Pagano and Turvey, 1998; Pagano and Day, 2020).

A body schema can change due to changes made to the body or to the body's ability, such as adding a tool to the body (Pagano and Day, 2020). For example, Gibson (1979/2014) uses the example of an individual using a pair of scissors, stating that "A tool is a sort of extension of the hand... and one can actually feel the cutting action of the blade" (p. 35; see Figure 1). When using a tool, we do not perceive the tool itself; rather, we perceive what the tool is doing in relation to our body, as such is the case with the scissors example. Moreover, tools alter the fit between the organism and their environment, which in turn alters the affordances available to the animal. The organism does not perceive these affordances as being performed by the tool independent of the user (meaning they do not perceive the behavior as being performed by the tool), rather, they perceive the affordances as actions they can perform by means of the tool (perceiving what the tool is doing rather than the tool itself).

The fluidity of body schemas suggests that animals are adaptive and sensitive to the changes their body can encounter. An alternative view to the traditional body schema (a collection of neural processes that determines the posture of one's body in space) is the 
embodied action schema, defined by Pagano and Day (2020) as representing "the body's current action capabilities, including any effects of a tool...” (p. 11). Evidence for this view is that both limbs and hand-held objects are perceived by detection of the same invariants (Pagano and Turvey, 1998). In other words, hand-held objects (i.e. tools) are perceived through the same process as one perceives their limbs, meaning the same information detected through the limbs is detected by means of the tool. This explains why body schemas are malleable and why attached objects, such as tools, can be incorporated into the body schema. This also explains why we perceive what the tool is doing rather than the tool itself.

\section{Learning in Tool Use}

In order for a tool to become a part of one's embodied action schema, sufficient practice (enough to bring about proficient use of a tool) is required to incorporate a tool into one's body schema. That is, proficient use is needed before one's awareness is of what the tool is doing rather than of the tool itself. The calibration to one's changed action capabilities with tools involves a specific mapping from embodied units of perception to embodied units of action (Proffitt and Linkenauger, 2013; Pagano and Day, 2020). This is consistent with a shift in focus from a traditional stable body schema to the embodied action schema, allowing for action capabilities to change and be modified with mastery of tools use (Pagano and Day, 2020).

One crucial aspect of learning is that it may not necessarily generalize across particular behaviors. If the behaviors do not share similar movements or goals, then the learned behaviors will not transfer to the new behavior. For example, infants must learn to perceive what slopes are safe for them to crawl down but this information does not transfer over to walking: infants that learn what slopes are unsafe to crawl down will attempt to walk down these unsafe slopes (Adolph, 2019). The behaviors are too dissimilar (the visual field in crawling versus walking is 
lower, the head and legs position in crawling is different than with walking, etc.) to generalize across the behaviors. This can also be seen with tools, such as SSDs (like the white cane). Blind users who had previously used a white cane had to go through the same training period as blindfolded participants to learn to use an auditory substitution device, the Voice (Auvray et al., 2007a). This suggests that the perceptions and behaviors involving the white cane and Voice were too dissimilar that even experienced SSD users had to learn to use the new device.

As tools become incorporated into the body schema (becoming an extension of oneself), tools can also become functionally transparent, meaning the user can feel the properties by means of the tool rather than (or in addition to) properties of the tool itself (Pagano and Day, 2020; Wagman, Hartling, \& Madison, 2020). Once sufficient practice has been performed with the tool, and the user feels proficient in using the tool to perform goal-directed behaviors, the tool becomes 'transparent' (Pagano and Day, 2020; Auvray and Myin, 2009). "Transparency” can be defined as the user being immersed in the task allowed by the tool (focusing on what the goal-directed behavior is) rather than being aware of manipulating the tool itself (Auvray and Myin, 2009). The example Auvray and Myin (2009) provide is the white cane used by those who are visually impaired: as the cane becomes more transparent with continual use (more practice), the tactile sensations felt through the cane become less noticeable as just sensations. Instead, the tactile sensations provide information to the user, such as how far away from an object they are or how dense the object is. 


\section{Ecological Interfaces}

When using a tool, the user can (and does) feel the interaction of the tool and their environment at the point of the tool (where the tool interacts with the environment). Moreover, tools can be used to explore the environment as a probe, making the tool functionally transparent (Wagman and Hajnal, 2014a, b; Wagman, Hartling, \& Mason, 2020; Pagano and Day, 2020). In such cases, the probe serves as a medium for haptic perception (active exploration of the environment), rather than as the object of perception. This is similar to vision: Visual perception allows for perception of distal environmental surfaces and properties through the medium of light; however, one's attention is usually on the task at hand rather than on the aspects of the light itself (Pagano and Day, 2020).

Ecological Interface Design (EID) is the design of interfaces between people and technology so that the technology can become functionally transparent to the user. It attempts to create a natural mapping (a connection) between the user's perceptual systems (visual, auditory, tactile) and what the device is doing. Users of these devices designed with the EID principle can more easily be trained to attune to the invariants and perform goal-directed behaviors while using the devices (such as with the ET). Since less complex tools, such as a white cane or screwdriver, can become functionally transparent, it can be assumed that more complex devices, like SSDs, can also achieve functional transparency, and this is the goal of EID - to make the device transparent for the user at the level achieved with haptic probing and haptic displays (Burns and Hajdukiewicz, 2004; Pagano and Day, 2020; Vicente and Rasmussen, 1990).

An example of EID can be seen in the field of laparoscopic surgery, or minimally invasive surgery. Altenhoff et al. (2017) and Long et al. (2016) designed an EID to measure haptic distance-to-break (DTB), an invariant specific to when manipulated bodily tissue is about 
to break. DTB is analogous to optical time-to-contact, which is specified by the rate of optical expansion of the surface texture as a surface approaches (Pagano and Day, 2020). Altenhoff et al. (2017) and Long et al. (2016) investigated DTB in a laparoscopic surgery stimulator, consisting of a laparoscopic tool attached to a torque motor and directed by computer software to simulate the tool being pressed into tissue causing it to become deformed and for it to break if deformed too far (the tissue tear). With only minimal training (10-15 minutes), novice participants were able to perform the tasks with high degrees of accuracy, and experienced surgeons improved their ability to detect DTB after the same training (Altenhoff et al., 2017; Long et al., 2016; Pagano and Day, 2020). This shows not only that training for perceptual attunement allows the user to reach (some level of) mastery of the device, it also shows that the device allowed the user to detect and act upon invariants in the environment.

EID holds the assumption that if direct perception is to occur in an unmediated setting, it can also be possible for direct perception to occur with artificial displays as long as the displays convey the same lawfully structured information specific to the affordances of the animalenvironment system (Pagano and Day, 2020). To provide this structured information, EID devices are designed to convey information in a way analogous to unmediated perception and action (Pagano and Day, 2020). For example, in SSD designs, users can experience a 'vibrotactile flow' analogous to optic flow if the device utilizes motors that provide vibrotactile feedback. This also aids in making the device functionally transparent for the user: by exploiting lawful relations in structured stimulation patters (i.e. optic flow), the devices are able to serve as a substitute medium for our senses. 


\section{Vibrotactile Flow in SSD}

The purpose of sensory substitution devices (SSDs) is to provide functionality to the user by providing information about the world and the behaviors afforded to the user by means of one modality (e.g. haptics, or active exploration) when another modality is unavailable (Favela, Riley, Shockley, \& Chemero, 2018). Some SSDs use motors to generate "vibrotactile flow" which is analogous to optic flow: It specifies affordances of surface layout in particular of ground-level objects (Favela et al., 2018; Cancar et al., 2013). Furthermore, SSDs have been shown to allow the user to perceive object properties (shape and size) in a way analogous to vision (de Paz et al., 2019). Through active exploration with an SSD, the user obtains knowledge of their surrounding environment. Eventually, the SSD becomes functionally transparent as there is a shift of focus from the perturbation of the hand to the objects appearing in the distant environment (Froese et al., 2008).

The SSD provides information to the user through vibrotactile stimulation; however, this stimulation only occurs if the user is actively scanning their environment. Devices like the "EyeCane", "Enactive Torch", and "TSIGHT" use infrared lasers to measure distance and then translate that information into vibrations (Maidenbaum et al., 2014; Favela et al., 2018; Cancar et al., 2013). If the user stops scanning the environment, or if the object is too far away, the device will not produce the necessary vibrations needed for perception. Although this might appear to be a disadvantage of SSDs, it actually models the natural process of spatial perception, such as tactile human exploration and whisking in animals (Maidenbaum et al., 2014). Moreover, the changing vibratory patterns that occur when using an SSD in a cluttered environment are comparable to the changing optical patterns that occur when visually perceiving in a cluttered environment. For example, objects that block the image interrupt perception similar to the 
sighted visual field (Auvray and Myin, 2009). If an object blocks our visual field, we can no longer see past the object, and this is the same with SSDs: if an object blocks the visual field of the device, the user can no longer 'see' past the object (see figure 6a and 6b). This means that in vision and when using an SSD, perception can be interrupted when an obstacle appears between the individual and the perceived object because the 'visual flow' (optic flow or vibrotactile flow) is interrupted.

A

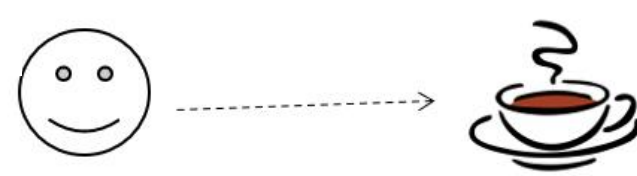

$\stackrel{\leftrightarrow}{4}$
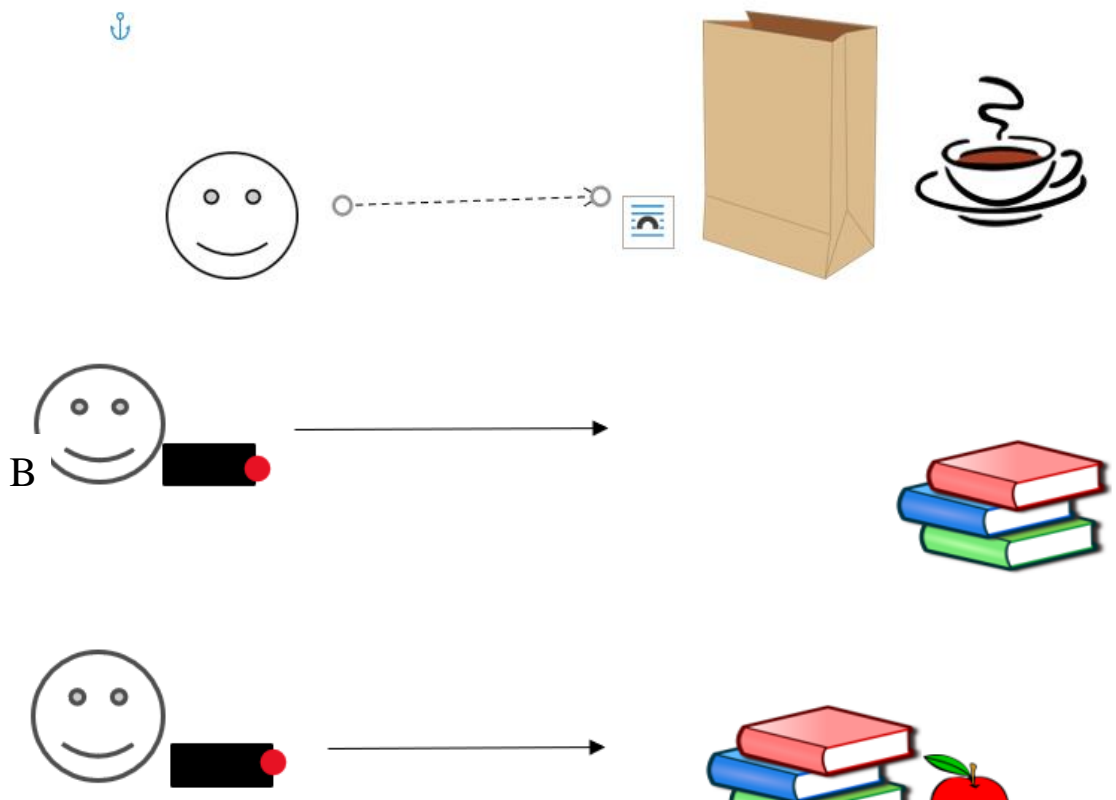

Figure $6 a$ and $6 b$ Obstruction of Visual Field ( $a$ ) and Obstruction of visual field with $\operatorname{SSD}(b)$ : When an object is placed in the way of something we are viewing, such as a paper bag in front of a coffee cup, we can no longer view the object because the visual field is blocked (a). Similar with an SSD: when an object is placed in front of another object, the device cannot detect the blocked object (b). 
Exploratory movements with SSDs provide information needed to perceive and act on affordances just as it does with exploratory visual behaviors. SSDs do this by providing the user with a vibrotactile flow equivalent to optic flow that the user can use to navigate and interact with their environment. A study conducted by Maidenbaum et al. (2012) explored how accurate their device, the "EyeCane", was at providing distance information to the user (when blindfolded) when using vibrotactile stimulation as the feedback for "seeing". The "EyeCane" translates point-distance information into auditory and tactile cues by using different auditory and tactile frequencies.

Their study had three experiments: experiment 1 tested the user's ability to perceive when using the device; experiment 2 tested the user's ability to navigate when using the device; and experiment 3 tested the user's ability to detect and avoid obstacles when using the device. The results showed that the participants were able to estimate, above chance level, the distance of a sheet of cardboard at varying distances (experiment 1), navigate through a winding corridor while gradually reducing the number of collisions with the wall (experiment 2), and avoid obstacles when they were added to the winding corridor (experiment 3). Furthermore, the control group that performed without the device (and still blindfolded) showed worse performance at the same tasks than those in the experimental groups using the device (Maidenbaum et al., 2014). Overall, the study provides evidence that varying frequencies of vibrotactile stimulation provide enough information to the user to determine the distance of objects and avoid collision with them. This also provides evidence that exploratory movements provide the user with a vibrotactile flow equivalent to optic flow that the user can use to navigate and interact with their environment. 
Other studies investigated more complex behaviors with SSDs, such as movement coordination with respect to approaching objects (like a soccer ball). Another device, "TSIGHT", uses distance-based information (like the EyeCane) to provide vibrotactile feedback to the user, allowing the perception of affordances (Cancar et al., 2013). The study found that users were able to perceive time of contact of an approaching ball at levels above chance, and that users were able to coordinate arm movements to hit the ball back to the experimenter. The success of the user to hit the ball back to the experimenter provides further evidence that users of SSD can perceive affordances using vibrotactile flow and can thus perform these complex behaviors, similar to how sighted individuals use optic flow. This can only occur, however, when there is continuous exploratory movement by the user, providing further evidence that vibrotactile flow is dependent on the exploratory movements of the user and the device. Their study provided evidence that users of SSDs can detect vibrotactile flow, suggesting that vibrotactile flow provides information just as optic flow does, such as time-to-contact of some objects (like the tossed ball).

\section{Sensory Substitution Devices as Tools}

SSDs are used primarily in experiments where the participant uses the device to perform goal-directed behavior. Tools are generally defined as extensions of the body that can be used to perform goal-directed behaviors, meaning that SSDs fit into this category. De Paz et al. (2019) conducted a study using a vibrotactile SSD to determine if participants could successfully determine if they could pass through a doorway when the doorway was scaled to their own shoulder width. The study found that the exploratory movements made with the device together with the vibrotactile stimulation were informative about the aperture width, and the participant used both means of information to perceive the passability of the aperture, meaning the user was 
able to detect the affordance of passing through the doorway by means of the device. A different study conducted by Favela et al. (2018) investigated participants' ability to use vision or an SSD to perceive affordances for passing through an aperture. The results suggested exploration of either the optic or vibrotactile stimulation was sufficient for the participants to perceive affordances for passing through an aperture.

The results of both studies suggest that exploratory movements with the SSD are a key feature in perceiving affordances when using the device (which is also the case in using a handheld probe to perceive affordances) (Wagman and Hajnal, 2014a, b; Wagman et al., 2020). In both studies, the participants were able to successfully perceive the relation between the width of the aperture and the width of their own body, suggesting that the device provides enough information for the participants to perceive possibilities for behavior with respect to their embodied action schema.

Lastly, the longer participants were allowed to practice with the device, the better they performed on the passability task, also suggesting that as the individual gets comfortable with using the SSD, the device becomes progressively functionally transparent. That is, their focus shifts from the device itself to the information it provides, as has been also found with tool use. In a study conducted by Grandon et al (2018), participants used the Enactive Torch to navigate through a maze. The study found that the longer the participant used the Enactive Torch, they no longer reported "feeling" the device, as if they had incorporated the device into their body schema. This suggests that, as individuals gain experience in using the device, they pay less attention to the device itself (the vibrations felt while wielding it) and more to the information the device is providing (what the vibrations mean, such as their location in the maze). While this example suggests that users of tools pay attention to the sensory input from the tool and they use 
this information in their body schemas, it also suggests that a learning period is needed before proficiency in a tool can be achieved. 
CHAPTER III: ADDRESSING CLAIM 1: SSDS IN PERCEPTION AND ACTUALIZING AFFORDANCES

This chapter will address claim 1, that users of SSDs can perceive and act on affordances to perform everyday behaviors, such as navigate among obstacles in their environment. Studies have shown that participants were able to use SSDs to perceive whether doorways could be passed through, and to navigate through an obstacle-maze (Favela et al., 2018; Froese et al., 2012). The chapter will describe the mechanics (i.e. how SSDs can be used to substitute one modality for another) of SSDs and examine the literature on the use of SSDs to provide further support for this claim. 


\section{Sensory Substitution Devices (SSDs) Review}

What a sensory substitution device is. To review, there are two different sensor mechanisms that an SSDs design can utilize: 1) an infrared laser that measures distances (Maidenbaum et al., 2014; Kerdegari et al., 2015; Cancar et al., 2013; Favela et al, 2018) and 2) a camera that creates a pixelated image of an object (Amith-Kumar et al., 2019; Bird et al., 2009 Stetten et al., 2007; Horvath et al., 2014). Both sensor mechanisms convert one form of energy (in this case light) into another, such as tactile vibrations (Cancar et al, 2013; Froese et al., 2012) or auditory output (Maidenbaum et al., 2014). Experiments with SSDs have shown that judgements about passing through a doorframe (Favela et al, 2018) and distance estimates to an object (Maidenbaum et al., 2014) were both significantly more accurate when participants used the SSD than with vision. The studies compared the use of an SSD, while blindfolded, to vision alone. Participants being more accurate with the SSD could be due to the participant's use of the device: participants may be more meticulous when using the device, making for more accurate usage of the device. Other studies have found that participants are able to successfully determine which behaviors they can perform while using SSDs, such as catching a ball or walking through a door frame (Bird et al., 2009, Favela et al., 2018). 
The purpose of these devices is to provide the user with information about the world by means of one modality when another modality is unavailable or compromised (such as in individuals who are visually impaired) so that the user can perceive and act within their environment. SSDs, then, allow the user to detect information about their environment that they might otherwise obtain by other means under other circumstances. This information provides the user with an awareness of their surrounding environment and the behaviors that can be performed within it. The SSD allows the user to perform these behaviors within their environment, meaning the user of the SSD perceives affordances with the device and can act upon those affordances by means of the tool (SSD). SSDs have shown that individuals are able to select from the many perceivable affordances, making the devices useful tools in studying behavior. Moreover, SSDs allow participants to perceive and act on the affordances as they would if they were using vision.

Sensory substitution devices in perception. SSDs allows the user to directly interact with (or perform goal-directed behaviors in) their environment, meaning the user of the SSD can perceive affordances and can act upon those affordances. Through active exploration of the environment, users of SSDs are able to use vibrotactile flow that is analogous to optic flow, providing environmental information such as time to contact with a surface. When using an SSD, individuals can detect fixed apertures, such as doorways, or dynamic ones, such as gaps, allowing for judgment of affordances such as step-over-able or step-through-able when using an SSD (Favela et al., 2018). Studies have even shown that in some cases, judgements made using an SSD are more accurate than those made by using vision (Favela et al, 2018; Maidenbaum et al., 2014). This provides evidence that SSDs allow the user to perceive and act on affordances in a way that is comparable to vision. 
Similarities of vision and SSDs. Similarities between the visual system and visual-totactile SSDs allow for SSDs to provide the analogous information as vision, thus allowing for the perception of affordances by means of the device. These similarities include 1) the formation of an image on a surface (retina for vision and lens of a camera for SSDs); 2) the presence of discrete elements on the surface that register the stimulation (rods and cones for vision, vibration nodes for SSDs); 3) the ability to actively explore the environment using movements comparable to movements of the eye and head (active exploration/scanning with the device); and 4) a similar pattern of occlusion when objects are located in front of or behind other objects (Morgan, 1977; Auvray and Myin, 2009).

Moreover, studies have shown increased activation of the visual cortex when using SSDs. Specifically, increased activation in V1 (of the primary visual cortex) after practice with vibrotactile SSDs. This suggests that the information obtained with the devices is similar to what would be obtained by vision and that the information is processed in the brain in an analogous way (Reiner et al., 2005a, Ptito and Kupers, 2005). The main differences between vision and SSDs seem to be quantitative rather than qualitative: SSDs have fewer receptors than vision (making the image resolution low) and movement of the camera on SSDs is limited compared to the visual system (Auvray and Myin, 2009). 
Mechanics of Sensory Substitution Devices. Haptic perception is perception of the body, objects attached to the body, and surfaces explored with the body or an attached object (perception through touch). The haptic system provides information about the relative position and orientation of the different body parts and the relative positions and orientations of objects with respect to the body (Carello and Turvey, 2004; Mauerberg-deCastro, et al., 2014). The use of an SSD (that provides vibrotactile or other mechanical information) to explore surrounding surface is analogous to the use of a long cane or a probe to perform the same task.

Studies using SSDs have shown that individuals are able to perceive obstacles in a maze and behavior-relevant features of their environment, such as edges (Horvath et al., 2013; Iannacci et al., 2011; Grandon et al., 2018). Individuals are also able to control behaviors, such as maintaining stable posture, through haptic exploration of the environment by means of direct or indirect contact with a support surface (by means of a tool) (Mauerberg-deCastro, et al., 2014). Active exploration allows the individual to position their body within the environment in the most efficient way. 
Tool use, such as with SSDs, directly mediates the relationship between the organism and the environment (Mauerberg-deCastro, et al., 2014). This allows the individual to use the tool as an extension of their body to assist in posture control. For example, an individual might use a walking stick or cane to maintain their balance when walking or hiking. SSDs also can be used to assist individuals in maintaining balance as the device provides more general spatial information about the environment. For example, individuals are able to use SSDs to navigate through a maze with minimal collisions with obstacles or walls (Favelas et al., 2018). Moreover, individuals are able to maintain the same stride speed when using the SSD as when using vision (Grespan et al., 2018). This suggests that the haptic information provides enough information for the individual to control the movements of their body. 


\section{Perception of environmental properties using SSDs}

Perception with SSDs provide access to analogous information about the surrounding environment as obtained through vision (light), only by different means. The camera and sensors on the SSD detect the differences in light much the same as the eyes detect differences in light in the visual field. However, in the SSD, the light information detected by the camera is further translated into a different modality (vibrations or audition) for the user. This information can be used to perceive particular properties of the environment or objects.

The camera and sensors detect environmental properties and then provide information to the user about these properties. The SSD detects differences in properties like edges and density of objects and translates the information into vibrations. In this way, the SSD can provide awareness of the layout of the environment for the user much like vision provides to a sighted individual. The devices described in the following sections have been used in experiments and have shown success in allowing users to perceive such properties.

Fingersight. This device has a miniature video camera mounted on the dorsal side of a particular finger that provides real-time image analysis of the environment, such as identifying particular features or objects. The image is then translated into vibrations via a small cell phone vibrator also attached to the dorsal side of the same finger (Stetten et al., 2007). The design of the device, however, limits the ability of the device to detect rudimentary features of objects and surfaces, such as simple edges. However, users of the Fingersight device are able to differentiate between angles as small as $15^{\circ}$, showing the device is still useful for perceiving subtle environmental properties (Horvath et al., 2013). With continual advancement of the camera used in the device, Fingersight could be used to perceive more complex features such as object identity (Stetten et al., 2007; Horvath et al., 2013). 
Haptic Laser. This device uses a laser pointer and a camera to detect features of the environment, and then translates this information into vibrotactile feedback using two vibration motors (Iannacci et al., 2011). The device encodes macro-textures (the density of objects) and physical distances of target objects and can detect edges. The device accomplishes this by modulating the vibrations to simulate various features of the environment. Surface texture is conveyed by the intensity of vibration. Coarse macro-texture is conveyed through more intense vibration feedback (similar to the feeling of dragging a rod across a surface). Distances are conveyed through shifts in the center of mass similar to a recoil-like sensation. Edges are conveyed by a sharp transition in the vibrations (Iannacci et al., 2011). A preliminary evaluation with the device by Iannacci et al. (2011) showed that the user was able to identify large transitions in the surface features of an unknown-to-them room (such as between walls) and identify clusters of objects.

Enactive Torch. This device is a distance-to-tactile perceptual system designed to investigate embodied action in the perception of spatial properties (Grespan et al., 2008). The Enactive Torch (Froese et al., 2012; Favela et al., 2018) is a flashlight sized sensory-substitution device that is held in one hand and connects to a vibrotactile motor (see figure 7). The Enactive Torch uses infrared range sensors to measure distances to adjacent surfaces/objects which are then translated to haptic stimuli (vibrations) by the motor. 
In a study by Grandon et al. (2018), participants were asked to navigate blindfolded through a room filled with obstacles and were asked to find their way as fast as they could while using the ET to avoid the obstacles. These results suggest that participants were able to navigate their environment and successfully avoid obstacles detected with the ET, suggesting SSDs like the ET can detect environmental properties (the obstacles) the user can then interpret. In addition, results showed that participants improved their performance over time with practice, suggesting that there is a learning component to using the ET effectively.

\section{Perception of affordances using SSDs}

Users of SSDs are able to use the vibrotactile feedback from the device to perform goaldirected behaviors such as walking from place to place. Users of the SSD have shown many of the same behavioral patterns, such as active exploration of the environment, as sighted individuals (Auvray and Myin, 2009), suggesting that the devices can be used comparably to perceive affordances.

TSIGHT. This device was developed to investigate the ecological proposal that individuals detect higher-order variables that specify the relationship between the animal and their environment (Gibson 1979/2014; Cancar et al., 2013). More specifically, it was used to investigate the proposal that vibratory stimulation on the skin can allow for perception of environmental properties such as time to contact (Cancar et al., 2013). The device was designed to allow for natural movement in exploration of real environments while providing vibrotactile feedback. The device uses a Kinect sensor (a camera with a depth sensor) to measure distances to surfaces and incorporates vibrotactile actuators for vibrotactile feedback (Cancar et al., 2013). 
In the experiment by Cancar et al. (2013), participants first used a computer (a virtual reality environment) to interact within a virtual environment to determine time to contact of an approaching virtual ball to use as a visual comparison. In the second experiment, participants used TSIGHT (while blindfolded) to determine when to hit an approaching ball. Participants were trained for a period of ten minutes during which the ball was repeatedly thrown to them. For the first 4 minutes they could use vision together with the device, and the rest of the time they were blindfolded and verbally informed of the moment of impact of the ball. The study found that participants were as successful in using TSIGHT to determine time to contact as they were using vision in the virtual environment. Furthermore, the study provides evidence that information about time to contact provided by 'vibrotactile flow' is analogous to information about to contact provided by optic flow.

EyeCane. The EyeCane was designed to improve on the traditional white cane through use of tactile (vibrations) and auditory (tones) output for detecting distances and angles. The goal of this device is to increase the user's spatial awareness of their environment by extending their ability to reach within the environment (Maidenbaum et al., 2014). The EyeCane device translates distance information into different auditory frequencies and tactile cues (vibrotactile feedback) through infrared emitters and sensors (motors) (Maidenbaum et al., 2014; Buchs, Simon, Maidenbaum, and Amedi, 2017). 
In the study conducted by Maidenbaum et al. (2014), participants attempted to navigate through a maze and avoid obstacles placed on the ground or walls by using the EyeCane, and they indicated aloud when they detected an obstacle. Obstacle clearance was defined as walking around or stepping over the obstacle. The study found that participants were able to successfully detect the obstacles and avoid them, even with minimal training with the device. This shows that participants were able to use the device to perceive affordances and avoid the obstacles in the maze, suggesting the EyeCane would be beneficial for the user to perceive and actualize affordances (such as to avoid obstacles) with a fast learning curve (Maidenbaum et al., 2014).

Enactive Torch. The study conducted by Favela et al. (2018) discussed in chapter 2 provides evidence that the ET can be used to perceive affordances. Participants using the ET were able to determine if they could successfully pass through an aperture (i.e., if they could perceive affordances for passing through the aperture with the ET). Moreover, the boundary between aperture widths judged as pass-through-able were no different with the ET and with vision, further suggesting the potential for the ET to enable perception of affordances.

\section{Summary}

SSDs are designed to provide information about the environment to the user when one modality is compromised (i.e. vision). The information provided to the user is similar (even analogous) to optic flow (i.e. vibrotactile flow) and provides the user information about their body in relation to the environment as well as the spatial layout of the environment. This information can then be used to perceive affordances and perform behaviors such as navigate through a maze or pass through a doorway. SSDs, then, can be used like vision to allow the user perception of goal-directed behaviors. 


\section{CHAPTER IV: ADDRESSING CLAIM 2: SSDS IN REHABILITATION}

The previous chapters have discussed the Ecological approach to perception and action and described tool use in perception. SSDs have also been discussed as tools that can be used in perception research. This chapter will focus on how ecological psychology and SSDs can be used to create 'functional therapeutics' that focus on the organism-environment relationship.

\section{A function-based approach to rehabilitation}

Functional Therapeutics. According to the International Classification of Functioning, Disability, and Health (ICF), rehabilitation requires the promotion of improvement of the patient's behavior/actions within relevant contexts. The main aim of rehabilitation should be to improve performance in functional tasks; however, the current state of rehabilitation lacks this direct approach. Currently, therapies focus on the neurophysiologic theories (a focus on cognitive, emotional, and/or behavioral deficits), thus isolating the organism from their environment. This approach limits the achievement of functional goals because the focus is on biological processes (a focus on micro-level pathology to macro-level social constraints), placing the emphasis on (and perhaps within) the organism only and thus excluding environmental properties that might play a role (Vaz et al., 2017). The ICF promotes a change to the way rehabilitation is viewed, with a shift from neurophysiological to the organism-environment system.

The ICF suggests the main focus of rehabilitating therapeutics should be to improve functionality of the organism, and to do so, the organism and its context (environment) must be considered together, not in isolation. This suggests a shift to an 'action-based therapy', also called 'functional therapeutics' (Vaz et al., 2017). This also promotes a shift in theory, from neurophysiological to perception and action, such as the ecological approach to perception and 
action from James J. Gibson (1979/2014). The focus of this approach is on organism-

environment mutuality, a conception the ICF encompasses, placing the emphasis on biological, individual, and social factors (Vaz t al., 2017).

Ecological Psychology as rehabilitation theory. Ecological psychology (a law-based theory of perception and action) provides an explanation for how perception informs and is informed by action, meaning this theory can provide an explanation for functional movement. Self-organization of functional behavior of living systems is a principle of ecological psychology that the ICF supports in rehabilitation therapeutics, suggesting that activity is shaped by nonlinear dynamics of the organism-environment system (Turvey et al., 1982; Vaz et al., 2017). According to the ecological perspective, information is specific to the state of the organismenvironment system allowing for regulation of action (Gibson, 1979/2014). This makes the organism-environment the unit of analysis for functional rehabilitation because the action of the organism is inescapably context-dependent (Vaz et al., 2017). The ecological approach, then, is consistent with the ICF model, and can provide a foundational principle for functional therapeutics.

Affordance focused therapeutics. As discussed in Chapter 1, affordances are opportunities for action and are dependent on the environment and the individual's specific capabilities. It is this relationship that is the framework for Ecological Psychology: A focus on the fit between the organism and its environment. From the Ecological perspective, then, the focus of rehabilitation should be to "restore or increase affordance-effectivity pairs for a patient" (changes in fit between animal and environment) through evaluation of the relevant environmental properties as they relate to the patients' capabilities (Vaz et al., 2017, pp. 126). This can be done through intrinsically-scaled or action-scaled measures. For example, a study done by Warren (1984) 
found that the perception of both maximum and optimal climb-able riser height was constrained by the relationship between leg length and rise height (rather than the height of the stair riser itself). Just as affordances depend on this fit between the action capabilities of the organism and its environment, so too should rehabilitation therapies.

The study by Warren (1984) shows that the context of actions (i.e. the action of stair climbing) is most usefully described with a metric that is related to the task-specific action (i.e. the relationship between leg length and riser height rather than riser height alone). The context is part of the action (meaning it is dependent on the environment and the organism). Rehabilitation currently focuses on the individual function outside of context, and this may not be the best practice for functionality of the patient. Instead, rehabilitation should scale properties of the environment and of the patient to provide the optimal level of challenge during therapy, and to obtain the optimal level of functioning (Vaz et al., 2017).

\section{Tool Use in Therapeutics}

Dynamic Touch. Ecological psychology as the theory for rehabilitation places the focus of therapy on the patient and their environment, meaning therapies would focus on functional behavior improvement for the patient. One area of investigation in Ecological psychologyhaptic perception via dynamic touch — offers a way for therapies to focus on the patientenvironment system. Haptic perception is "the awareness of the body and the environment by means of the body (i.e. mechanical contact)" and dynamic touch is "the awareness of magnitudes and directions of implements and limbs by means of muscular effort" (Carello et al., 2008, pp 340). In other words, organisms can perceive affordances of wielded objects or by means of wielded objects (e.g., by using a white cane). Dynamic touch exploits the physics of rotation and mechanical invariants specific to relations between the body and hand-held objects. Information 
about relevant relationships is provided through exploratory movements with the object (Carello et al., 2008). In therapy, dynamic touch would offer patients more coordinated and efficient movement patterns through increased awareness of different possibilities for behavior provided by means of wielded objects (Carello et al., 2008; Vaz et al., 2017).

Perception by means of a wielded object is dependent on the mechanical properties of the object — primarily its (the objects) mass distribution (Carello et al., 2008). This sensitivity to the object's mass distribution can be used in rehabilitation therapies. Carello et al. (2008) suggests training patients to explore and use objects with varied mass distributions to increase their awareness of diverse opportunities the objects provide for performing a task. Training with diverse mass distributions may enable the user to generalize learned abilities across objects of like capabilities and circumstances. The idea of using therapy to attune patients to movementbased constraints (based on dynamic touch) can be applied to SSDs as well. SSDs are not typically brought into mechanical contact with environmental surfaces, but they are typically wielded by muscular effort, so the devices do utilize the same movement-based constraints suggested by Carello et al. (2008).

Dynamics of SSDs. Dynamic touch exploits the physics of rotation and mechanical invariants to perceive properties of the hand-held object and the environment (by means of the object). This is similar to how SSDs exploit different modalities (by utilizing touch or hearing) to provide information (such as visual information through this other modality) to the user to perceive properties of the environment. Although SSDs do not come into mechanical contact with a surface, SSDs do provide an awareness of the body and the environment through active exploration by means of the device (a vibrotactile feedback), much the same as a tool would provide. 
External objects (i.e. tools), such as a gripper or stepping stool, can be used to modify an individual's action capabilities and therefore modify what affordances are perceivable. In the case of SSDs, these devices can be used to modify action capabilities and therefore modify what affordances are perceivable. As already discussed in the previous chapters, the studies conducted by Maidenbaum et al. (2014) and Cancar et al. (2013) provided evidence that users of SSDs can perceive and act upon affordances to avoid obstacles on a navigation course and to hit a ball that was tossed to them by substituting visual information about surfaces and objects for tactile information about those surfaces and objects. The user was able to use the vibration frequencies as distance information about such surfaces and objects and thereby avoid obstacles or coordinate arm movements for action when their vision was impaired (blindfolded). The SSD provided the user with a 'vibrotactile flow' that in turn provided information about the relationship between the user and the environment. This information was obtained through active exploration with the device, much in the same way as an individual might use a tool such as a long cane, providing a 'pseudo-dynamic touch'. That is, the device does not provide haptic feedback about the environment, but it does provide feedback by substituting haptics for another modality (such as vibrations).

\section{SSDs outside of laboratory research}

SSDs for visual rehabilitation. SSDs are non-invasive interfaces that have been shown to convey information about relationships to objects and surfaces by converting light information into another form (such as tactile or auditory; see chapter 3). Research with these devices has suggested the devices could be used as aids for those visually impaired, providing perceptual capabilities (such as perception of shape and location) the user might otherwise not have available. Moreover, research also suggests the devices would be a possibility for a non-invasive 
rehabilitation technique (Maidenbaum et al., 2014). In 95\% of people who are visually impaired, the brain parts needed for vision (occipital cortex) are still intact (the problem being with the eye itself), suggesting that these brain parts can be stimulated by other means, such as in sensory substitution (Maidenbaum et al., 2014). The information detected by SSDs can be conveyed to the brain via the visual pathways even if the information is tactile vibrations (Maidenbaum et al., 2014; Elli et al., 2014).

SSDs are non-invasive aids that aim to assist those who are visually impaired (regardless of their level of blindness) through behavioral compensation and reorganization of the brain (Elli et al., 2014). Studies have shown that SSDs recruit the occipital cortex and other brain regions to process information independent of the sensory modality by which information is obtained (meaning if the information is gained haptically, it is processed in the visual pathway as visual information) (Amedi et al., 2007; Reich et al., 2012; Elli et al., 2014). Using SSDs, then, seems to activate the same brain regions as vision, allowing the user the ability to use these brain regions in perceiving affordances that might otherwise be perceived by vision.

Problems with SSDs adoption into rehabilitation. The difficulty in adopting any given SSDs for medical usage is the device itself: it must be small, lightweight, and inexpensive enough to manufacture for users (Maidenbaum et al., 2014; Favela et al, 2018). Many individuals suffering from visual impairments (e.g., blindness) shy away from using long canes as aids because of the stigma of having them, as well as their large design (Maidenbaum et al., 2014). Many researchers have taken these issues into account when designing their SSDs, even if their intention is not necessarily for rehabilitation use. Even for use in research, it would be beneficial to have a small, lightweight, and inexpensive device to use. 
Another consideration is the ergonomics of SSDs, such as the potential problem of compromising the user's ability to use other perceptual systems. For example, devices that require the use of headphones limits the hearing ability of the individual because other sounds may be muffled. Instead, auditory information might be transmitted to the cochlea through bone conduction (Elli et al., 2014). SSDs might also interfere with other coping strategies or adaptive information processing individuals have developed on their own (Elli et al., 2014; Maidenbaum et al., 2014).

Research using SSDs in rehabilitation therapies. SSDs are currently used mainly as research tools in psychology to study perception and action behaviors; however, the original intent for the devices was to be used for visual rehabilitation (Maidenbaum et al., 2014). Paul Bach-y-Rita developed a device called the 'Tactile Vision Sensory Substitution' (TVSS) that allowed visually impaired users to complete a variety of tasks such as recognize large letters and catch a ball when tossed to them (Bach-y-Rita et al., 1969). The design of the TVSS incorporated motors that vibrated to provide the user with tactile information about their environment (visionto-tactile sensory substitution). The development of the TVSS suggested that these devices could provide visual information by touch (or audition), showing the devices to be useful in noninvasive rehabilitation for those visually impaired (Maidenbaum et al., 2014).

Some studies have already adopted the mechanisms (vibrotactile feedback) behind SSDs into their research for rehabilitation methods. For example, a study analyzing post stroke hemiplegia patients incorporated vibrotactile stimulation to alert patients that the toe of their foot collided with a virtual object (Jaffe et al., 2004). In this study, participants walked on a treadmill while wearing virtual reality helmets that presented them with a virtual obstacle course (while on the treadmill). The course consisted of visible virtual objects that the participants were asked to 
step over. If the participant collided with a virtual object, a tone sounded and a vibrotactile stimulus was applied to their heel or toe (from a motor attached to them in a boot) to alert them of the collision. The visual and vibrotactile feedback were found to improve participants walking strides and (virtual) object clearance (Jaffe et al., 2004). In this study, the vibrotactile feedback served as a sensory substitution for a virtual collision. A computer system detected when a participant's foot intersected with the virtual object and would then trigger the 'foot switch' (vibrator attached to the participants foot) to vibrate and alert the participant of this intersection/collision. Although this study does not use an SSD directly, the results provide evidence that SSDs could be beneficial for patients suffering from motor impairments.

The study by Jaffe et al. (2004) provides evidence that vibrotactile feedback can work in a rehabilitation setting, with patients improving their walking strides (longer stride length), walking speed (an increase in walking speed) and object clearance (increased clearance between toe and object) when given vibrotactile, auditory, and visual feedback information. Another study using the concept of vibrotactile feedback found that vibrating soles assist in balance control. In a study by Priplata et al., (2003) young and elderly participants stood on vibrating insoles and the researchers measured sway parameters and random-walk variables. The sway parameters were the parameters of postural sway when standing still (how far the individual's body swayed when standing still) and random 'walk' (or random moving of the foot) variables were defined as moving the foot as if to walk (moving the foot from the standing position). The study found the vibrating insoles to assist the elderly participants more than the young participants in mediolateral range and critical mean square displacement. The study suggests that noise- and touch-based devices, like the vibrating gel-insoles, could assist those suffering from motor impairments (as well as visual impairments). 
Another study by Maidenbaum et al. (2013) used their device, the EyeCane, to investigate the accessibility of the device for those visually impaired by using a virtual environment to learn how to use an SSD (the Virtual EyeCane). Maidenbaum et al. (2013) reformatted the EyeCane to be a virtual tool the participants were able to use while navigating a virtual maze, much like a videogame controller. The Virtual-EyeCane, like the physical device, uses sensors to detect objects from a distance and transforms the information into auditory information (a series of beeps, with closer objects triggering higher frequencies). The study utilized blind and sighted blindfolded participants, asking the participants to navigate through a virtual twisting maze using only the Virtual-EyeCane. The study found that all participants were able to use the device to navigate the virtual environment, suggesting that the device aided in navigating an environment the participant otherwise would not have been able to (Maidenbaum et al., 2013). Moreover, the results also suggest that virtual environments could be beneficial to train users of SSDs in, because it offers a risk-free way to learn how to use the device (in a real world environment, the user could collide with real objects and get injured).

\section{Summary}

Ecological psychology focuses on the animal-environment system; specifically, affordances (based on action capabilities in relation to their environment). This approach should be adopted into rehabilitation therapies to set the focus of therapy on the individual's functionality and move away from neurophysiological centered therapies. One way to utilize and improve functionality for individuals is with SSDs. SSDs have shown beneficial effects in experiments investigating perception and action; however, this is only utilizing one aspect the devices offer. The devices offer feedback to the user based on another sense that they have intact 
(auditory or tactile), suggesting the devices could be used by those visually or motor impaired to improve their daily functioning. 


\section{CHAPTER V: EVALUATION OF THE ENACTIVE TORCH AND FUTURE DIRECTIONS}

\section{The Enactive Torch}

The ET is a handheld device designed on the principles of the enactive approach to interface design, meaning that goal-directed actions are at the center of the functionality of the device (Favela et al., 2018). The ET (Froese et al., 2012; Favela et al., 2018) is a "flashlight sized" sensory-substitution device (length: $15.8 \mathrm{~cm}$; width: $5.8 \mathrm{~cm}$; height: $4.6 \mathrm{~cm}$; weight: 350 g) that is held in one hand and connects to a vibrotactile motor. The vibrotactile motor is connected to the hand-held torch by a wire embedded in the wrist strap. The Enactive Torch uses infrared range sensors to detect distances to adjacent surfaces/objects (with a range of 0.1 to $1 \mathrm{~m}$ ) which are then translated to haptic stimuli (vibrations) by the motor. The vibration intensity is inversely proportional to the distance of the surface detected by the device: a smaller range sensor can detect distances of 8-80 cm and larger range sensors can detect distances of 20-150 cm (Favela et al., 2018). By sweeping the Enactive Torch back and forth the user obtains information about the layout of nearby surfaces according to the time-varying patterns of vibrations of changing intensity (Froese et al., 2012; Favela et al., 2018).

\section{The fit of the ET for rehabilitation}

The ET device itself is not the focal point of the devices' design (meaning the device was not designed around the technology piece itself); rather, it was designed for the user's ability to perform behaviors by means of the device. Many SSDs were designed with the purpose of functionality, but the ET was specifically designed to limit the amount of technology the device utilized. Devices like the EyeCane (Maidenbaum et al., 2014) use a large band of motors for vibrotactile feedback whereas the ET uses a single motor attached to the wrist (Favela et al., 2018; Forese et al., 2012). According to Maidenbaum et al.'s (2014) evaluation of SSDs 
limitations, the "clunkiness" of a device deters individuals from using them (because they are not discrete), so reducing the number of motors used (and thus reducing the size of the device) makes the ET a more appealing and functional design for visually impaired users.

ET and functional therapeutics. The ET detects distance information through infrared light (or sonar) and translates this information into vibrations felt on the wrist of the user. Users of the ET are able to perceive affordances such as pass-through-able-ness of an aperture (Favela et al., 218) and are able to navigate through environments when blind-folded (Froese et al., 2012; see chapter 3). This provides evidence that the device is well suited for supporting performance of goal-directed behaviors and functional mobility, making the device useful in functional therapeutics because the user does not experience the device but rather what they can do with it (Favela et al., 2018). Other devices, such as the EyeCane (Maidenbaum et al., 2014) have also been shown to support goal-directed behaviors and functional mobility; however, there have been more studies conducted using the ET, suggesting this device is more 'standardized' for use outside of the laboratory.

The vibrations from the ET convey distance information to the user based on the inverse relationship of vibration intensity and distance, but the vibrations also give information about the objects themselves. The detection of objects works the same way as the detection of distance: The ET sensors' receptor field is shaped like a cone, giving an 'inverse shadow' effect, meaning the further an object, the larger of a shadow it will cast (see figure 7; Grespan et al., 2008). A study by Grespan et al. (2008) showed that the ET is sensitive to size information sufficient for object recognition. The study used one small $(3 \mathrm{~cm})$ and two medium-sized $(9 \mathrm{~cm})$ cylindrical objects and one large $(32 \mathrm{~cm})$ flat object. The study found that participants used the size of the object to determine how far away it was, using a sort of algorithmic procedure (Grespan et al., 
2008). This finding shows that the ET not only detects distance but also more definitive details like object size and shape.

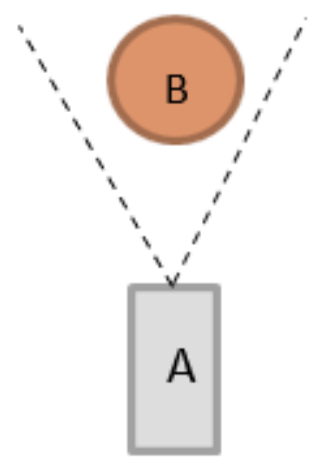

Figure 7 Inverse Shadow Effect: The receptor field for the ET is like a cone shape, resulting in an 'inverse shadow' effect where the further an object, the larger it appears because it stays in the receptive field longer.

The ability to use the ET to perceive both distance and object information makes it a useful tool for functional therapeutics. Functional therapeutics has a focus on the individual's ability to perform goal-directed behaviors (Vaz et al., 2017). The ability of the ET to detect specific information about objects gives more information about the fit between the user and their environment, meaning that the ET enables the user to potentially perceive more affordances for more behaviors (since it can detect more information about the environment). While other devices, like the EyeCane (Maidenbaum et al., 2014) and Fingersight (Horvath et al., 2014) allow detection of edges, the ET has been shown to be more accurate in whole object detection and recognition (Grespan et al., 2008). 


\section{Future Directions}

Improved Training. Studies have shown that individuals with little training using SSDs were able to detect affordances (such as pass-through-able, Favela et al., 2018) and navigate through mazes (Froese et al., 2012; González Grandon et al., 2018). Studies showing that the ET can detect distance and object properties also suggests that the devices might be modified to detect details such as shading or color, prompting more intensive training of the basic functions of the device and eventually a more dynamic training for more dynamic behaviors (Grandon et al., 2008; Maidenbaum et al., 2014). Training should also be more extensive, such as having dedicated modules for specific real-life functions (like recognition of faces and everyday objects) the user will encounter while using the device (Maidenbaum et al., 2014).

Moreover, virtual training (in a virtual environment) should be incorporated into the training regimen just as often as in-person training (in a real environment) One reason is that virtual training provides the user the ability to familiarize themselves with the device in a safe environment (such as with the Virtual EyeCane, Maidenbaum et al., 2013; Auvray and Myin, 2009; Jaffe et al., 2004). Another reason is to counterbalance the cost and effort involved with more intense training regimes: virtual environments allow the user to train at their own pace and can be done anywhere, limiting the number of personnel needed for training (Maidenbaum et al., 2014). Lastly, virtual environments "gamify" the training, and thus have the possibility to increase the users' motivation to learn how to use the device (Maidenbaum et al., 2014; Auvray and Myin, 2009).

Real-world training environments should also be simplified so the user can become accustomed to using the device in a noisier environment. This can be done with either 1) a dedicated "safe environment" in the real-world where training would take place, or 2) dedicated 
virtual environments that augment the real-world, or 3) an input-simplification algorithm that filters out input beyond a certain range to limit the "noise" the user experiences while training (Maidenbaum et al., 2014). Virtual environments would allow the user a safe area to practice using the device and get accustomed to how the device worked, but real-world training environments (or simulated real-world) would be needed for the user to get accustomed to using it in real-world, noisy environments (where they will be primarily used).

Active Sensing. As previously discussed, affordances are perceived through active exploration and interaction with the environment (Gibson, 1979/2014). SSDs should be designed so that an active sensory-motor loop is continuously provided to the user (Maidenbaum et al., 2014). In many research studies, users lose this active sensory-motor loop (due to the highly controlled laboratory environment), causing them to lose information about their environment. With this continual feedback, behavior would improve as the user would be provided a continual feedback about their environment.

Modules for the SSD. The three modules currently comprising SSDs are 1) a processing unit (extracts data to generate a representation), 2) input sensors (capture visual information), and 3) output human machine interface (portrays the data) (Maidenbaum et al., 2014). Future designs should attempt to design the software for these modules to improve integration algorithms and machine learning techniques to filter the information detected. This would assist in alleviating the mental load for the user and simplify the device for use (Tan et al., 2010; Lescal et al., 2013; Maidenbaum et al., 2014). 


\section{In Conclusion}

SSDs have been primarily used in research, but the devices also have the (current) ability to be used in rehabilitation for those with visual or motor impairments. SSDs utilize the enactive interface design, focusing on the user being able to perform goal-directed behaviors. The ET is the most "standardized" of the SSDs due to its extensive research, and for this reason, should be considered for rehabilitation therapies. Future directions should improve the algorithms of the device to assist in information filtration, and training regimens should be designed to be more dynamic and interactive for the user for better results when using an SSD. 


\section{REFERENCES}

Adolph, E. K. (2019). An ecological approach to learning in (not and) development. Human Development. DOI: 10.1159/000503823

Altenhoff, N. M., Pagano, C. C., Kil, I., and Burg, T. C. (2017). Haptic Distance-to-Break in the Presence of friction. Journal of experimental psychology: Human Perception and Performance, 43, 231-244.

Amedi, A., Stern, W. M., Camprodon, J. A., Bermpohl, F., Merabet, L., Rotman, S. and PascualLeone, A. (2007). Shape conveyed by visual-to-auditory sensory substitution activates the lateral occipital complex, Nat. Neurosci. 10, 687-689.

Auvray, M. and Myin, E. (2009). Perception with compensatory devices: From sensory substitution to sensorimotor extension. Cognitive Science, 33, 1036-1058

Bach-y-Rita, P., Collins, C.C., Saunders, F. A., White, B., and Scadden, L. (1969). Vision Substitution by Tactile Image Projection, Nature, vol. 222 (5184), pp. 963-964

Bird, J., Marshal, P., \& Rogers, Y. (2009). Low-fi skin vision: A case study in rapid prototyping a sensory substitution system. In: Proceedings of the 2009 British Computer Society Conference on Human-Computer Interaction, 1-5 Sep 2009, UK, pp. 55-64

Buchs, G., Simon, N., Maidenbaum, S., and Amedi, A., (2017). Waist-up protection for blind individuals using the EyeCane as a primary and secondary mobility aid. Restorative Neurology and Neuroscience, 35, 225-235

Burton, A. and Radford, J. (1978). Thinking in Perceptive: Critical Essays in the Study of Thought Processes. Routledge. ISBN 978-0-416-85840-2

Burns, C. M. and Hajdukiewicz, J. R. (2004). Ecological Interface Design. New York: CRC Press. 
Cancar, L., Diaz, A., Barrientos, A., Travieso, D., and Jacobs, M. D. (2013). Tactile-Sight: A sensory substitution device based on distance-related vibrotactile flow. International Journal of Advanced Robotic Systems

Carello, C., Silva, P. L., Kinsella-Shaw, J. M., \& Turvey, M. T. (2008). Muscle-based perception: theory, research and implications for rehabilitation. Brazilian Journal of Physical Therapy, 12(5), 339-350.

Carello, C., \& Turvey, M.T. (2004). Physics and psychology of the

Muscle sense. Current Directions in Psychological Science, 13, 25-28. doi:10.1111/j.09637214.2004.01301007.x

Cole, G. W., Chan, L. Y. G., Vereijen, B., Adolph, E. K. (2013). Perceiving affordances for different motor skills. Exp Brain Res, 225, 309-319. Doi/:10.1007/s00221-012-3328-9

De Paz C., Travieso D., Ibáñez-Gijón J., Bravo, M., Lobo, L., \& Jacobs, D. M. (2019). Sensory substitution: The affordance of passability, body-scaled perception, and exploratory movements. PLoS ONE, 14:3, e0213342

Elli, V. G., Benetti, S., and Collignon, O. (2014). Is there a future for sensory substitution outside academic laboratories? Multisensory Research, 27, 271-291

Favela, H. L., Riley, A. M., Shockley, K., \& Chemero, A. (2018). Perceptually equivalent judgments made visually and via haptic sensory-substitution devices. Ecological Psychology, 30:4, 326-345. doi:10.1080/10407413.208.1473712

Froese, T., McGann, M., Bigge, W., Spiers, A., \& Set. K. A., (2012). The Enactive Torch: A new tool for the science of perception. IEEE Transactions on Haptics, 5, 365-375. doi:10.1109/TOH.2011.57 
Gibson, J. J. (2014) The ecological approach to visual perception: classic edition. Psychology Press. (Original work published in 1979)

Gibson, P. (1979). The Vivaldi Aerial. 1979 9th European Microwave Conference, 101-105. https://doi.org/10.1109/EUMA.1979.332681

González Grandon, X.A., Zapata Fonseca, L.I., Gómez Escobar, H., Ortíz Garín, G.U., Flores Mares, J.J., Sáenz Burrola, A.A., \& Froese, T. (2018). The enactive torch: Interactive embodied learning with a sensory substitution Interface

Grespan, L., Froese, T., Di Paolo, A. E., Seth, K. A., Spiers, A., \& Bigge, W. (2008). Investigating the role of movement in the constitution of spatial perception using the Enactive Torch. In: ENACTIVE08

Head, H. (1920). Studies in Neurology, Vol. 2. London,: Oxford University Press.

Horvath, S., Galeotti, J., Wu, B., Klatzky, R., Siegel, M., and Stetten, G., (2014). FingerSight: Fingertip Haptic Sensing of the Visual Environment. IEEE Journal of Translational Engineering in Health and Medicine, vol. 2.

Iannacci, F., Turnquist, E., Avrahami, D., and Patel, N. S., (2011). The haptic laser: MultiSensation tactile feedback for at-a-distance physical space perception and interaction. Resented at CHI 2011, May 7-12, 2011, Vancouvor, BC, Canada

Iodice, P., Scuderi, N., Saggini, R., and Pezzulo, G. (2015). Multiple timescale of body schemareorganization due to plastic surgery. Human Movement Science, 42, 54-70

Ishak, S., Adolph, K. E., and Lin, C. G. (2008). Perceiving affordances for fitting through aperatures. Journal of experimental psychology: Human perception and performance. 34 (6), 1501-1514 
Jaffe, L. D., Brown, A. D., Pierson-Carey, D. C., Buckley, L. E., \& Lew, L. H. (2004). Stepping over obstacles to improve walking in individuals with post stroke hemiplegia. Journal of Rehabilitation Research \& Development, 41(3A), 283-292

Kerdegari, J., Kim, Y., and Prescott, T. (2015). Tactile language for a head-mounted sensory augmentation device. In: Biometric and Biohybrid Systems, Lecture Notes in Computer Science (9222)., pp 359-365. ISBN 978-3-319-22978-2

Lescal, D., Rouat, J., and Voix, J. (2013). Sensorial substitution system from vision to audition using transparent digital earplugs. In: Proceedings of Meetings on Acoustics, p. p040014

Long, L. O., Pagano, C. C., Singapogu, R. B., and Burg, T. C. (2016). Surgeon's perception of soft tissue constraints and distance-to-break in a simulated minimally invasive surgery task. Proceedings of the human factors and ergonomics society 2016 annual meeting, 1598-1602. Washington, DC, September 19-23, 2016

Maidenbaum, S., Abboud, S., and Amedi, A. (2014). Sensory substitution: Closing the gap between basic research and widespread practical visual rehabilitation. Neuroscience and Biobehavioral Reviews, 41: 3-15

Maidenbaum, S., Hanassy, S., Abboud, S., Buchs, G., Chebat, D-R., Levy-Tzedek, S., \& Amedi, A. (2014). The "Eyecane", a new electronic travel aid for the blind: Technology, behavior, \& swift learning. Restorative Neurology and Neuroscience, 32, 813-824

Maidenbaum, S., Levy-Tzedek, S., Chebat, D-R., and Amedi, A. (2013). Increasing Accessibility to the blind of virtual environments, using a virtual mobility aid based on the "EyeCane": Feasibility Study 
Mark, L. (1987). Eye height-scaled information about affordances: A study of sitting and stair climbing. Journal of Experimental Psychology:, 13(3), 361-370. https://doi.org/10.1037/0096-1523.13.3.361

Mauerberg-deCastro, E., Moraes, R., Paioli Tavares, C., Andreeta Figueiredo, G., C.M. Pacheco, S., and Costa, D. A. T. (2014). Haptic Anchoring and human postural control. Psychology and Neuroscience, 7 (3), 301-318

Morgan, M. J. (1977).Molyneux's question. Vision, touch and the philosophy of perception. Cambridge, England: Cambridge University Press.

Pagano, C. C., and Turvey, M. T. (1998). Eigenvectors of the inertia tensor and perceiving the orientation of limbs and objects. Journal of experimental psychology: Human perception and performance, 40, 404-415

Pagano, C. C., \& Day, B. M. (2020). Ecological interface design inspired by "The meaningful environment”. In J. B. Wagman \& J. J. C. Blau (Eds.) Perception as the Detection of Information: Reflections on Gibson's Ecological Approach to Visual Perception. (pp. $37-$ 50). New York: Routledge

Ptito, M., \& Kupers, R. (2005). Cross-modal plasticity in early blindness. Journal of Integrative Neuroscience, 4, 479-488

Priplata AA, Niemi JB, Harry JD, Lipsitz LA, Collins JJ. Vibrating insoles and balance control in elderly people. Lancet. 2003 Oct 4; 362(9390):1123-4. Doi: 10.1016/S01406736(03)14470-4. PMID: 14550702.

Proffitt, D. R., and Linkenauger, S. A. (2013). Perception viewed as a phenotypic expression. W. In Prinz, M. Beisert and A. Herwig (Eds.) Action Science: Foundations of an emergency discipline. Cambridge, MA: MIT Press. 
Reich, L., Maidenbaum, S. and Amedi, A. (2012). The brain as a flexible task machine: implications for visual rehabilitation using noninvasive vs. invasive approaches, Curr. Opin. Neurol. 25, 86-95.

Renier, L., Collignon, O., Poirier, C., Tranduy, D., Vanlierde, A., Bol, A., Veraart, C., \& De Volder, A. G. (2005a). Cross-modal activation of visual cortex during depth perception using auditory substitution of vision. NeuroImage, 26, 573-580.

(13) (PDF) Perception with Compensatory Devices: From Sensory Substitution to Sensorimotor Extension.

Royden, C. S., and Moore, K. D., (2012). Use if speed cues in the detection of moving objects by moving observers. Vision Research, 59: pp. 17-24, doi:10.1016/j.visres.2012.02.006

Stetten, G., Klatzky, R., Nichol, B., Galeotti, J., Rockot, K., Zawrotny, D., Sendgikoski, N., and Horvath, S., (2007). Fingersight: Fingertip Visual Haptic Sensing and Control. In HAVE 2007 - IEEE International Workshop on Haptic Audio Visual Environments and their Application

Tan, S. S., Maul, T. H. B., Mennie, N. R., Mitchell, P. (2010). Swiping with luminophonics. In: IEEE Conference on Cybernetics and Intelligent Systems (CIS), pp. 52-57

Thomas, J. B., Riley, A. M., and Wagman, J. B. (2020). Information and Its Detection. In J. B. Wagman \& J. J. C. Blau (Eds.) Perception as the Detection of Information: Reflections on Gibson's Ecological Approach to Visual Perception. (pp. 130-148). New York: Routledge

Turvey, M. T. (2019). Lectures on perception. An ecological perspective. New York: Routledge. 
Turvey, M. T., Fitch, H. L., \& Tuller, B. (1982). The Bernstein perspective: I. The problems of degrees of freedom and context-conditioned variability. In J. A. S. Kelso (Ed.), Human motor Behavior: An introduction (pp. 239-252). Hilsdale, NJ: Lawrence Erlbaum Associates.

Vaz, D. V., Silva, P. L., Mancini, M. C., Carello, C., \& Kinsella-Shaw, J. (2017). Towards an ecologically grounded functional practice in rehabilitation. Human Movement Science, $52,117-132$.

Vincente, K. J. and Rasmussen, J. (1990). The ecology of human-machine systems II: Mediating direct perception in complex work domains. Ecological Psychology, 2, 20-249.

Wagman, J. B. (2020). A guided tour of Gibson's Theory of Affordances. In J. B. Wagman \& J. J. C. Blau (Eds.) Perception as the Detection of Information: Reflections on Gibson's Ecological Approach to Visual Perception. (pp. 130-148). New York: Routledge

Wagman, B, J, Cialdella, T. V., and Stoffregen, A. T. (2018). Higher order affordances for reaching: Perception and performance. Quarterly Journal of Experimental Psychology, 112. Doi: $10.1177 / 17470218784403$

Wagman, B. J. and Hajnal, A. (2014). Getting off on the right (or left) foot: Perceiving by mean of a rod attached to the preferred or non-preferred foot. Exp Brain Res, 232, 3591-3599. Doi: 10.1007/s00221-014-4047-1

Wagman, J. B., \& Morgan, L. L. (2010). Nested Prospectivity in perception: Perceived maximum reaching height reflects anticipated changes in reaching ability. Psychonomic bulletin \& review, 17(6), 905-909. 
Wagman, B. J., Hartling, S., and Mason, J. J. (2020). Selective perception in probing by foot: Perceiving the length of a probe and the distance of a probed surface. Acta Psychologica, 209

Warren, H. D., and Strelow, R. E. (1985). Electronic Spatial Sensing for the Blind: Contributions from Perception. Springer, ISBN 978-90-247-2689-9

Warren, W. H. (1984). Perceiving affordances: visual guidance of stair climbing. Journal of Experimental Psychology Human Perception and Performance, 10(5), 683-703. 